\title{
Diversidade dos Braconidae (Hymenoptera) da Unidade de Conservação de Teixeira Soares, Marcelino Ramos, RS, com ênfase nos Microgastrinae
}

\author{
Rozane Maria Restello \& Angélica Maria Penteado-Dias²
} 'Programa de Pós-Graduação em Ecologia e Recursos Naturais, Universidade Federal de São Carlos, Caixa Postal 676, 13565-905
São Carlos-SP, Brasil. rrozane@uri.com.br
${ }^{2}$ Depto. de Ecologia e Biologia Evolutiva, Universidade Federal de São Carlos, Caixa Postal 676, 13565-905 São Carlos-SP, Brasil.
angelica@power.ufscar.br

\begin{abstract}
Braconidae diversity (Hymenoptera) from the Unidade de Conservação Teixeira Soares, Marcelino Ramos, RS, with emphasis on Microgastrinae. Three Malaise traps were used, respectively, in a degrading area, native wood area and gallery forest at the Unidade de Conservação Teixeira Soares, Marcelino Ramos, RS, from November, 1999 to December, 2000. A total of 2,442 braconid specimens were collected distributed in 23 subfamilies, most of them coming from gallery forest. Many genera constitute new occurrences to Rio Grande do Sul State, Brazil. Microgastrinae, Rogadinae, Opiinae and Helconinae were the most abundant groups, representing $78.3 \%$ of the total. The 885 Microgastrinae specimens were identified and distributed into 28 genera. Glyptapanteles was the most common in all sampled points, followed by Apanteles and Diolcogaster. Indexes of occurrence and dominance, diversity and equitability are also presented.
\end{abstract}

KEYWORDS. Bio-indicator; Brazil; Malaise trap; parasitoids.

RESUMO. Diversidade dos Braconidae (Hymenoptera) da Unidade de Conservação de Teixeira Soares, Marcelino Ramos, RS, com ênfase nos Microgastrinae. Neste estudo utilizamos três armadilhas Malaise coletando, respectivamente, em área degradada, mata mesófila e mata ciliar da Unidade de Conservação Teixeira Soares, Marcelino Ramos, RS, de novembro de 1999 a dezembro de 2000. Foram coletados 2.442 espécimes de Braconidae distribuidos em 23 subfamílias, a maioria proveniente da mata ciliar. Muitos dos gêneros constituem nova ocorrência para o Estado do Rio Grande do Sul, Brasil. Microgastrinae, Rogadinae, Opiinae e Helconinae foram os grupos mais abundantes, representando 78,3\% do total. Foram identificados 885 espécimes de Microgastrinae, distribuídos em 28 gêneros. Glyptapanteles foi o mais comum em todos os pontos amostrados, seguido por Apanteles e Diolcogaster. Índices de ocorrência e dominância, diversidade e equitabilidade são também apresentados.

PALAVRAS-CHAVE. Armadilha Malaise; bioindicador; Brasil; parasitóides.

Os Hymenoptera Parasitica representam o grupo de maior riqueza de espécies dentro de Hymenoptera, tanto que Egleton (1990) destaca que a sua diversidade é grande e que a necessidade de coletas de material tem precedido às observações etológicas. Os Braconidae incluem 34 subfamílias e representam a segunda maior família de Hymenoptera, com aproximadamente 40.000 espécies no mundo (Wharton et al. 1997).

Os insetos parasitóides constituem o maior componente de muitos ecossistemas terrestres com mais de $20 \%$ de todas as espécies, portanto, a avaliação da diversidade deste grupo apresenta grande relevância (Lasalle \& Gould 1993, Godfray 1994) e apesar da sua abundância, pouco se sabe sobre a sua estrutura de comunidade, especialmente nos trópicos (Lasalle 1993). González \& Burgos (1997) comentam a importância de se conhecer a diversidade desses insetos bem como a sua distribuição em áreas da região Neotropical, para desta forma estabelecer as bases do conhecimento necessário frente a sua importância em trabalhos de controle biológico, através da utilização dos inimigos naturais.

Em De Santis (1980) há 27 citações de Braconidae para o Rio Grande do Sul, incluindo algumas espécies de Microgastrinae.
Segundo Whitfield \& Lewis (1999) e González \& Ruiz (2000), os Braconidae podem ser utilizados como grupo indicador do grau de preservação dos ecossistemas pela estimativa da riqueza de espécies existentes em uma determinada região e para o controle de insetos-praga para a agricultura minimizando o uso de agroquímicos.

Os Microgastrinae constituem a maior subfamília dentre os Braconidae, com mais de 2500 espécies com distribuição mundial, ocorrendo praticamente em todos os habitats (Shenefelt 1972, 1973, Mason 1981, Gauld \& Bolton, 1988 e Whitfield 1995).

Os objetivos deste estudo foram estudar a fauna de Braconidae, com ênfase em Microgastrinae, da Unidade de Conservação Teixeira Soares, Marcelino Ramos, RS, além de comparar a sua diversidade em áreas com vegetação em diferentes estágios de conservação.

\section{MATERIAL E MÉTODOS}

Área de estudo: a Unidade de Conservação Teixeira Soares, localiza-se ao norte do estado do Rio Grande do Sul, no município de Marcelino Ramos, a 27²8'9" de latitude Sul e $51^{\circ} 54$ '5" Oeste, próxima a foz do Lajeado Teixeira Soares, na 
margem esquerda do rio Uruguai. Possui 469,32 hectares e segundo Veloso Goés \& Filho (1982), situa-se em região de transição entre a Floresta Estacional Decidual e a Floresta Ombrófila Mista. Apresenta relevo entalhado, com alguns trechos de alta declividade. A média anual de temperatura é de $18,7^{\circ} \mathrm{C}$, subindo de agosto a janeiro e entrando em declínio em fevereiro até julho, com probabilidade de geada de abril a setembro (outono-inverno) (Socioambiental, 2000). A precipitação média anual é de $1708 \mathrm{~mm}$, bem distribuídos, não havendo grande concentração de períodos chuvosos ou secos.

As armadilhas Malaise foram montadas na borda dos seguintes ambientes naturais em diferentes estágios de conservação:

Área degradada: com cobertura vegetal alterada por ação antrópica (pastagens, plantio de feijão e milho). A cobertura vegetal caracteriza-se por várias espécies de gramíneas e de espécies de maior porte como: Andropogon bicornis H. B.\& $\mathrm{K}$. (capim-rabo-de-burro), Rhynchelytrum repens (Willd.) Hubbard (capim-natal), Chloris sp (capim-coqueiro) (Poaceae). Algumas espécies arbustivas como Solidago microglossa DC. (erva-lanceta), Vernonia tweediana Baker (mata-pasto) (Asteraceae) formam o estrato mais alto.

Mata mesófila: a mais bem preservada, com sucessão vegetal, dependendo do local observado, variando de intermediária a avançada e onde ocorrem: Solanum erianthum D. Don (fumão) (Solanaceae), Schinus terbinthifolius Raddi (aroeira -vermelha) (Anacardiaceae), Allophylus edulis (St. Hil) Ex Warm (chal-chal) (Sapindaceae); Nectandra grandiflora Nees \& Mart. Ex Nees (canela-amarela) (Lauraceae); Campomanesia xanthocarpa O.Berg. (guabiroba), Eugenia uniflora Linn. (pitangueira) (Myrtaceae). O solo acumula uma grande quantidade de folhiço e poucas poáceas.

Mata ciliar: à margem do Lageado Teixeira Soares, com área de drenagem de $96,4 \mathrm{Km}^{2}$, extensão de $29 \mathrm{Km}$. Nela predominam plantas de porte médio, principalmente os sarandis (Calliandra). Área com um processo de regeneração natural que varia de intermediária a avançada; o solo com grande quantidade de folhiço e numerosas poáceas. Ocorrem ainda espécies exóticas da família Zingiberaceae, além de árvores frutíferas, uma vez que no entorno havia antigas habitações.

Amostragem e identificação dos exemplares: foram realizadas capturas quinzenais, no período de novembro de 1999 a dezembro de 2000 com três armadilhas Malaise (Townes 1972).O material capturado foi transportado até o Museu Regional do Alto Uruguai da URI -Universidade Regional Integrada - Campus de Erechim/RS, para triagem onde os Braconidae foram separados e preservados em álcool $70 \%$ para posterior montagem em alfinetes entomológicos.

A identificação em níveis de subfamílias e gêneros de Microgastrinae, foi realizada com base em Mason (1981) e Wahl \& Sharkey (in Goulet \& Huber 1993).

Parte do material coletado foi depositado na Coleção Entomológica do MuRAU (Museu Regional do Alto Uruguai da URI-Campus de Erechim) e parte no Departamento de Ecologia e Biologia Evolutiva da Universidade Federal de São Carlos, SP (DCBU).
Análise dos dados : índices de ocorrência e dominância das subfamílias foram obtidos pelo método proposto por Palma (1975) apud Abreu \& Nogueira (1989). Utilizou-se o Índice de Shannon para estimar a diversidade de subfamílias e gêneros e o Î́ndice de Equitabilidade para avaliar a uniformidade da captura ao longo das amostragens (Magurran 1988).

\section{RESULTADOSE DISCUSSÃO}

Foram obtidos 2442 exemplares de Braconidae, a maior parte capturada na mata ciliar (1424 exemplares), seguida pela área de mata mesófila (601) e finalmente área degradada, com menor captura (417) (Tabela 1). Juillet (1960) observou que existe uma tendência à uma maior atividade dos Braconidae onde a vegetação é mais aberta, portanto a fisionomia do local parece ter influenciado na captura dos mesmos. O próprio curso d'água funciona como uma trilha aberta na vegetação, onde o movimento dos insetos é maior. De acordo com dados fornecidos pela EMBRAPA - Trigo de Passo Fundo/ RS, durante a realização deste estudo, a temperatura média foi $23^{\circ} \mathrm{C}$ e a umidade relativa do ar $71 \%$. Juillet (1964), observou que os Braconidae preferem temperaturas entre $20-24^{\circ} \mathrm{C}$ e umidade relativa do ar em torno de $75 \%$.

O número de subfamílias coletadas (23) corresponde a 67,6\% do total de Braconidae registrados para o Novo Mundo (34 subfamílias) (Wharton et al. 1997). As três armadilhas capturaram braconídeos de 23 subfamílias com hábitos cenobiontes e idiobiontes (Tabelas I e II). Os Microgastrinae (Tabela I) foram os mais freqüentemente coletados, incluindo espécies de 28 gêneros (Tabela III). Das subfamílias amostradas, 17 são comuns aos três pontos de coleta: Agathidinae, Alysiinae, Braconinae, Cardiochilinae, Cenocoelinae, Cheloninae, Doryctinae, Euphorinae, Gnamptodontinae, Helconinae, Homolobinae, Macrocentrinae, Microgastrinae, Miracinae, Opiinae, Orgilinae e Rogadinae, classificadas como endoparasitóides cenobiontes ou ectoparasitóides idiobiontes de ovos, larvas e pupas de Lepidoptera, Coleoptera e Diptera (Wharton et al. 1997). Microgastrinae, Rogadinae, Opiinae e Helconinae foram os grupos mais abundantes, representando cerca de $78,3 \%$ do total capturado nos três ambientes. Destes, Microgastrinae apresentou maior freqüência de ocorrência na amostragem total $(51,19 \%)$. Trata-se de grupo com espécies solitárias ou gregárias endoparasitóides cenobiontes de larvas de Lepidoptera. Observase um gradiente crescente na freqüência de ocorrência tanto para idiobiontes como cenobiontes da área degradada para a área de mata ciliar (Tabela II).

A fauna de Braconidae na região estudada é predominantemente ( $92 \%$ ) de hábitos cenobiontes (Tabela II) e endoparasitóide e tem como hospedeiros principais as larvas de Lepidoptera (Tabela I). Os Braconidae mais abundantes são os Microgastrinae $(\mathrm{N}=99)$ associados com hospedeiros herbívoros sobre folhagens (Mason 1981; Wharton et al.1997).

A mata ciliar distingue-se das demais pelas boas condições de recuperação das espécies vegetais originais sendo o ponto em que se obteve espécimes de 21 subfamílias das quais apenas uma é exclusiva (Adeliinae). 
Tabela I. Estratégia de parasitismo ( $\mathrm{C}=$ cenobionte; $\mathrm{I}=$ idiobionte ), hábito ( $\mathrm{S}=$ solitário; $\mathrm{G}=$ gregário), hospedeiros, número de exemplares capturados de Braconidae e frequência relativa em três pontos na Unidade de Conservação de Teixeira Soares, Marcelino Ramos/RS, no período de Novembro de 1999 a Dezembro de 2000.

\begin{tabular}{|c|c|c|c|c|c|c|c|c|}
\hline Subfamília & $\begin{array}{c}\text { Estratégia } \\
\text { Reprodutiva }\end{array}$ & Hábito & Hospedeiros & $\begin{array}{c}\text { Área } \\
\text { degradada }\end{array}$ & $\begin{array}{l}\text { Mata } \\
\text { mesófila }\end{array}$ & $\begin{array}{l}\text { Mata } \\
\text { ciliar }\end{array}$ & $\begin{array}{l}\text { Total } \\
\text { (n) }\end{array}$ & $\begin{array}{r}\text { Frequência } \\
\text { Relativa }\end{array}$ \\
\hline Adeliinae & $\mathrm{C}$ & $\mathrm{S}$ & Lepidoptera & 0 & 0 & 1 & 1 & $0,04 \%$ \\
\hline Agathidinae & $\mathrm{C}$ & $\mathrm{S}$ & Lepidoptera & 4 & 5 & 4 & 13 & $0,53 \%$ \\
\hline Alysiinae & $\mathrm{C}$ & $\mathrm{S} / \mathrm{G}$ & Diptera & 18 & 3 & 23 & 44 & $1,80 \%$ \\
\hline Aphidiinae & $\mathrm{C}$ & $\mathrm{S}$ & Homoptera & 1 & 0 & 8 & 9 & $0,37 \%$ \\
\hline Blacinae & $\mathrm{C}$ & $\mathrm{S}$ & Coleoptera & 3 & 0 & 13 & 16 & $0,66 \%$ \\
\hline Braconinae & I & $\mathrm{S} / \mathrm{G}$ & Lepidoptera, Coleoptera & 27 & 27 & 53 & 107 & $4,38 \%$ \\
\hline Cardiochilinae & $\mathrm{C}$ & $\mathrm{S}$ & Lepidoptera & 2 & 2 & 3 & 7 & $0,29 \%$ \\
\hline Cenocoelinae & $\mathrm{C}$ & $\mathrm{S}$ & Coleoptera & 2 & 1 & 4 & 7 & $0,29 \%$ \\
\hline Cheloninae & $\mathrm{C}$ & $\mathrm{S}$ & Lepidoptera & 6 & 13 & 9 & 28 & $1,15 \%$ \\
\hline Doryctinae & I & $\mathrm{S} / \mathrm{G}$ & Lepidoptera, Coleoptera & 19 & 21 & 33 & 73 & $2,99 \%$ \\
\hline Euphorinae & $\mathrm{C}$ & $\mathrm{S} / \mathrm{G}$ & $\begin{array}{l}\text { Coleoptera, Hymenoptera, } \\
\text { Neuroptera, Heteroptera, Psocoptera }\end{array}$ & 2 & 7 & 19 & 28 & $1,15 \%$ \\
\hline Gnamptodontinae & $\mathrm{C}$ & $\mathrm{S}$ & Lepidoptera & 6 & 9 & 5 & 20 & $0,82 \%$ \\
\hline Helconinae & $\mathrm{C}$ & $\mathrm{S}$ & Coleoptera & 12 & 34 & 102 & 148 & $6,06 \%$ \\
\hline Homolobinae & $\mathrm{C}$ & $\mathrm{S}$ & Lepidoptera & 2 & 10 & 6 & 18 & $0,74 \%$ \\
\hline Hormiinae & I & $\mathrm{S}$ & Lepidoptera & 0 & 5 & 13 & 18 & $0,74 \%$ \\
\hline Ichneutinae & $\mathrm{C}$ & $\mathrm{S}$ & Symphyta, Lepidoptera & 1 & 1 & 0 & 2 & $0,08 \%$ \\
\hline Macrocentrinae & $\mathrm{C}$ & $\mathrm{S} / \mathrm{G}$ & Lepidoptera & 5 & 8 & 5 & 18 & $0,74 \%$ \\
\hline Meteorinae & $\mathrm{C}$ & $\mathrm{S} / \mathrm{G}$ & Coleoptera, Lepidoptera & 0 & 1 & 0 & 1 & $0,04 \%$ \\
\hline Microgastrinae & $\mathrm{C}$ & $\mathrm{S} / \mathrm{G}$ & Lepidoptera & 182 & 302 & 766 & 1250 & $51,19 \%$ \\
\hline Miracinae & $\mathrm{C}$ & $\mathrm{S}$ & Lepidoptera & 11 & 8 & 14 & 33 & $1,35 \%$ \\
\hline Opiinae & $\mathrm{C}$ & $\mathrm{S}$ & Diptera & 38 & 76 & 62 & 176 & $7,21 \%$ \\
\hline Orgilinae & $\mathrm{C}$ & $\mathrm{S}$ & Lepidoptera & 8 & 17 & 62 & 87 & $3,56 \%$ \\
\hline Rogadinae & $\mathrm{C}$ & $\mathrm{S} / \mathrm{G}$ & Lepidoptera & 68 & 51 & 219 & 338 & $13,84 \%$ \\
\hline Total & & & & 417 & 601 & 1424 & 2442 & $100,00 \%$ \\
\hline
\end{tabular}

Segundo a classificação de Palma (1975 apud Abreu \& Nogueira ,1989) Microgastrinae e Rogadinae são consideradas comuns; Braconinae, Doryctinae e Miracinae como intermediárias e Agathidinae, Cardiochilinae e Cenocoelinae como raras nos três pontos de coleta.

O índice de diversidade de Shannon para as subfamílias foi semelhante para todos os habitats amostrados: 1,32 para a mata ciliar e 1,30 para área degradada e mata mesófila . O índice de Equitabilidade (J) variou de 57\% (mata ciliar) a 66\% (área degradada) sugerindo média equitabilidade e que a comunidade de Braconidae das áreas amostradas tende a coexistir com alguma dominância de determinados táxons.

Estrutura Taxonômica da Comunidade de Microgastrinae: Dos 1250 exemplares de Microgastrinae coletados apenas as fêmeas (885 exemplares) puderam ser identificados em 28 gêneros. A mata ciliar, foi a que apresentou a maior riqueza, com 28 gêneros, sendo 6 exclusivos deste local. A área de mata mesófila e a área degradada, apresentaram 15 gêneros, em ambas, nenhum exclusivo. Foram encontrados nove gêneros comuns aos três ambientes. Destes, a maioria é endoparasitóide cenobionte, utiliza ovos e principalmente larvas expostas de primeiros ínstares de Lepidoptera, sendo considerados parasitóides especialistas.

Os gêneros com maior número de exemplares capturados na mata ciliar (Tabela III) foram: Glyptapanteles Ashmead
1904 ( $\mathrm{n}=100)$ endoparasitóides de larvas de Macrolepidoptera, incluindo os Pyraloidea (Mason 1981); Apanteles Foerster, $1862(n=56)$ endoparasitóides generalistas de hospedeiros brocadores de caules e minadores de folhas das ordens Lepidoptera, Coleoptera e Diptera (Mason 1981); Diolcogaster Ashmead, $1900(\mathrm{n}=45)$ com espécies que são parasitóides solitários ou gregários de larvas de Macrolepidoptera (Noctuidae, Geometridae e Pyraloidea) (Mason 1981) e Deuterixys Mason, 1981 ( $\mathrm{n}=43)$ solitários, endoparasitóides cenobiontes de larvas de Bucculatrigidae e Lyonetiidae (Wharton \& Sharkey 1998). Na área de mata mesófila (Tabela III), os gêneros dominantes foram: Apanteles $(\mathrm{n}=54)$, Diolcogaster $(\mathrm{n}=19)$, Glyptapanteles $(\mathrm{n}=20)$ e Hypomicrogaster Ashmead, $1898 \quad(\mathrm{n}=17)$ que são endoparasitóides de larvas minadoras de Microlepidoptera (Mason 1981).

Tabela II. Número de exemplares e freqüência de idiobiontes e cenobiontes por ponto de coleta na Unidade de Conservação de Teixeira Soares, Marcelino Ramos/RS, no período de Novembro de 1999 a Dezembro de 2000.

\begin{tabular}{lccccc}
\hline Pontos & Total & Idiobiontes & $\%$ & Cenobiontes & $\%$ \\
\hline Mata Ciliar & 1424 & 99 & 7 & 1325 & 93 \\
Mata Mesófila & 601 & 53 & 8 & 558 & 92 \\
Área Degradada & 417 & 46 & 11 & 371 & 89 \\
\hline
\end{tabular}


Tabela III. Gêneros e $n^{\circ}$ de exemplares de Microgastrinae amostrados nos três pontos de coleta na Unidade de Conservação de Teixeira Soares, Marcelino Ramos/RS, no período de Novembro de 1999 a Dezembro de 2000 .

\begin{tabular}{|c|c|c|c|c|}
\hline Gêneros & $\begin{array}{c}\text { Área } \\
\text { degradada }\end{array}$ & $\begin{array}{c}\text { Mata } \\
\text { mesófila }\end{array}$ & $\begin{array}{l}\text { Mata } \\
\text { ciliar }\end{array}$ & Total \\
\hline Alphomelon & 1 & 5 & 2 & 8 \\
\hline Apanteles & 17 & 54 & 56 & 127 \\
\hline Clarkinella & 0 & 1 & 20 & 21 \\
\hline Cotesia & 15 & 10 & 28 & 53 \\
\hline Deuterixys & 11 & 6 & 43 & 60 \\
\hline Diolcogaster & 14 & 19 & 45 & 78 \\
\hline Distatrix & 2 & 0 & 6 & 8 \\
\hline Dolichogenidea & 0 & 0 & 6 & 6 \\
\hline Glyptapanteles & 32 & 20 & 100 & 152 \\
\hline Hypomicrogaster & 22 & 17 & 30 & 69 \\
\hline Iconella & 10 & 0 & 20 & 30 \\
\hline Illidops & 0 & 3 & 2 & 5 \\
\hline Larissimus & 0 & 0 & 1 & 1 \\
\hline Microplitis & 0 & 0 & 8 & 8 \\
\hline Papanteles & 0 & 1 & 1 & 2 \\
\hline Parapanteles & 1 & 3 & 16 & 20 \\
\hline Pholetesor & 0 & 1 & 18 & 19 \\
\hline Prasmodon & 0 & 2 & 9 & 11 \\
\hline Promicrogaster & 7 & 2 & 35 & 44 \\
\hline Protapanteles & 0 & 2 & 6 & 8 \\
\hline Protomicroplitis & 0 & 0 & 2 & 2 \\
\hline Pseudapanteles & 2 & 0 & 9 & 11 \\
\hline Sendaphne & 2 & 0 & 13 & 15 \\
\hline Snellenius & 1 & 0 & 25 & 26 \\
\hline Venanides & 3 & 0 & 9 & 12 \\
\hline Venanus & 0 & 0 & 1 & 1 \\
\hline Xantapanteles & 0 & 0 & 8 & 8 \\
\hline Xenogaster & 0 & 0 & 3 & 3 \\
\hline TOTAL & 182 & 302 & 766 & 1250 \\
\hline
\end{tabular}

Na área degradada (Tabela III) os mais comuns foram: Glyptapanteles $(\mathrm{n}=32)$, Hypomicrogaster $(\mathrm{n}=22)$, Apanteles $(\mathrm{n}=17)$ e Cotesia Cameron 1891(n=15), este último endoparasitóide solitário ou gregário de muitos macrolepidópteros (Mason 1981; Penteado-Dias 1986; 1987; Braga et al. 2001).

Os gêneros comuns aos três ambientes (Tabela III) foram: Glyptapanteles, Apanteles, Diolcogaster, Cotesia, Deuterixys, Promicrogaster Brues y Richardson, 1913, Alphomelon Mason 1981, Parapanteles Ashmead 1900 e Hypomicrogaster. Os índices de Diversidade de Shannon e de Equitabilidade para os gêneros desta subfamília, foram altos $\left(\mathrm{H}^{\prime}=4,06 \mathrm{e} \mathrm{J}=85,5 \%\right)$ para a mata ciliar comparados com aqueles da área degradada e mata nativa $\left(\mathrm{H}^{\prime}=3,2\right.$ e 2,9; J = $83,5 \%$ e $75 \%$, respectivamente). A área degradada apresenta grande poder de regeneração e favorece a alta diversidade de espécies de plantas possíveis nutridoras de grande diversidade de hospedeiros. A mata ciliar apresentou distribuição mais uniforme dos indivíduos entre os táxons.

Para Hawkins et al. (1992) conhecer a riqueza dos Braconidae de uma determinada área de estudo pode ser um indicador do estado de conservação das suas comunidades vegetais e de seus fitófagos. Os dados aqui apresentados indicam que na Unidade de Conservação de Teixeira Soares em Marcelino Ramos, RS a comunidade de Braconidae tem características que além de manter o equilíbrio dinâmico dos ecossistemas onde ocorre, também proporciona benefícios diretos aos agroecossistemas do entorno através do potencial controle de insetos pragas.

Agradecimentos. À URI - Campus de Erechim, pelo apoio logístico e financeiro, às bolsistas de Iniciação Científica Fernanda Viero Dias e Taymara Bonissoni pelo auxílio nos trabalhos de campo e laboratório, à Denise Scatolini e Paloma H. F. Schimabukuro pela ajuda na identificação dos Braconidae e a José Roberto Verani e Magda Yamada pelo auxílio nas análises estatísticas.

\section{REFERÊNCIAS}

Abreu, P. C. O. V. \& C. R. Nogueira. 1989. Spatial distribution of Siphonophora species at Rio de Janeiro Coast Brazil. Ciência e Cultura 41: 897-902.

Braga, S. M. P.; M. M. Dias \& A. M. Penteado-Dias. 2001. Aspectos bionômicos de Eois tegularia (Guenée) e Eois glauculata (Walter) (Lepidoptera, Geometridae, Larentiinae) e seus parasitóides. Revista Brasileira de Zoologia 18: $837-840$.

De Santis, L. 1980. Catalogo de los Himenopteros Brasileños de la Serie Parasitica incluyendo Bethyloidea. Editora da Universidade Federal do Paraná, Curitiba. 395p.

Eggleton, D. 1990. Male reproductive behavior of parasitoid wasp Lytarmes maculipennis (Hymenoptera: Ichneumonidae). Ecological Entomology 15: 357-360.

Fuchs, T. W.; F. R. Huffman \& J. W. Smith. 1979. Introduction and establishment of Apanteles flavipes. (Hymenoptera, Braconidae) on Diatraea saccharalis (Lepidoptera, Pyralidae) in Texas. Entomophaga 24: 109-114.

Gauld, I. D. \& B. Bolton. 1988. The Hymenoptera. Oxford: Oxford University Press. 332p.

Godfray, H. C. J. 1994. Parasitoids: Behavioral and Evolutionary. Ecology. Princeton: Princeton University Press. 473p.

González, H. D. \& F. A. L. Burgos. 1997. Gêneros de Braconidae (Hymenoptera) em Yucatan: alguns elementos para el plateamiento de patrones de riqueza. Acta Zoologica Mexicana 70: 65-77.

González, H. D. \& D. B. Ruíz. 2000. Los Braconidos (Hymenoptera: Braconidae) como grupo parâmetro de biodiversidade em las selvas decíduas del tropico: uma discusion acerca de su posible uso. Acta Zoologica Mexicana 79: 43-56.

Hawkins, B. A.; M. R. Shaw \& R. R. Askew. 1992. Relation among assemblage size, host Sopecialization, and Climatic Variability in North American parasitoids Communities. American Naturalist 139: $58-79$.

Juillet, J. A. 1960. Some factors influencing the flight activity of Hymenoptera parasites. Canadian Journal of Zoology 38: $1057-1061$.

Juillet, J. A. 1964. Influence of weather on flight activity of parasitic Hymenoptera Canadian Journal of Zoology 2: 1133-1141.

Lasalle, J. \& F. D. Gauld. 1993. Hymenoptera and Biodiversity. C. A. B. International $348 \mathrm{p}$.

Magurran, A. E. 1988. Ecological diversity and is measurement. Princeton, New Jersey: Princeton University Press. 179p.

Mason, W. R. M. 1981. The polyphiletic nature of Apanteles Foerster (Hymenoptera: Braconidae): a phylogeny and reclassification of Microgastrinae. Memoirs of the Entomological Society of Canada 115: 1-147.

Penteado - Dias, A. M. 1987. Contribuição para o conhecimento da morfologia e biologia de Cotesia alius (Mues., 1958) (Hymenoptera, Braconidae, Microgastrinae). Revista Brasileira de Entomologia 31: 439-443. 
Penteado-Dias, A. M. 1986. Parasitismo de Ascia monuste orseis (Latreille, 1919) (Lepidoptera, Pieridae), por Cotesia glomerata (L.1758) (Hymenoptera, Braconidae Microgastrinae). Revista Brasileira de Entomologia 30: 257-259.

Shenefelt, R. D. 1972. Braconidae 4, Microgastrinae: Apanteles. In: J. V. D. Vech; R. D. Shenefelt (Eds.). Hymenopterorum Catalogus (new edition), part 9. Junk, The Hague. p. 669-812.

Shenefelt, R. D. 1973. Braconidae 5, Microgastrinae, Ichneutinae. In: J. V. D. Vecht; R. D. Shenefelt (Eds.). Hymenopterorum Catalogus (new edition), part 9. Junk, The Hague. p. 669-812.

Socioambiental. 2000. Consultores Associados. Plano de Manejo da Unidade de Conservação da UHE-Itá Teixeira Soares/RS. Florianópolis/SC. 90p.

Townes, H. 1972. A light-weight Malaise trap. Entomological News 83: 225-229.

Veloso, P. H. \& L. Goés Filho. 1982. Fitogeografia Brasileira. Classificação fisionômica - ecológica da vegetação neotropical.
Boletim Técnico Projeto RADAMBRASIL. Série Vegetação. $85 \mathrm{p}$.

Wahl, D. B. \& M. J. Sharkey. 1993. Superfamily Ichneumonoidea. In: Goulet, H. J. T. Huber. Hymenoptera of the world: An identification guide to families. Ottawa: Research Branch Agriculture Canada Publication, p. 358-509.

Wharton, R. A.; P. M. Marsh \& M. J. Sharkey (Eds.). Manual of the New World Genera of the Family Braconidae (Hymenoptera). Washington: Special Publication of the International Society of Hymenopterists 1: 1-439.

Whitfield, J. B. 1995. Annoted checklist of the Microgastrinae of North America North of Mexico (Hymenoptera: Braconidae). Journal of the Kansas Entomological Society 68: 245-262.

Whitfield, J. B. \& C. N. Lewis. 1999. Analytical survey of braconid wasps fauna (Hymenoptera: Braconidae) on six Midwestern U.S. tallgrass prairies. Annals of the Entomological Society of America 94: 231-238.

Recebido em 02/05/2005; aceito em 08/02/2006 